\title{
"Comparison of Flexural Strength of two Provisional Materials Used In Fixed Prosthodontics -An in Vitro Study".
}

\author{
Dr. Dhanashree Beedkar, Dr. Mithilesh Dhamande, Dr. S.R. Godbole. \\ Department of Prosthodontics, Crown and Bridge, Sharad Pawar Dental College, \\ Datta Meghe Institute Of Medical Sciences, Wardha, India.
}

\begin{abstract}
Provisional prosthesis are the restorations that provide interim protection, mastication, esthetics and positional stability while the definitive restorations are being fabricated.

Flexural strength is an important mechanical property that determines the long term prognosis of provisional restoration. Although provisional prosthesis are used for short period of time, flexural strength of provisional material cannot be neglected. This study was an attempt to determine flexural strength Of commercially available autopolymerising material (DPI) and heat cure material (DPI) at the time interval of 24 hours of fabrication and after 7 days of fabrication.

The sample size was 15 samples of each material. Sample was subjected for 3 point flexural test. Within the limitations of the study flexural strength study of heat cure resin was significantly higher when compared to autopolymerising resin.
\end{abstract}

Keywords: Autopolymerising resin, Flexural strength, Heat cure resin, Provisional prosthesis, 3Point flexural test.

\section{Introduction}

Rehabilitation of partially edentulous patients using Fixed Partial Denture (FPD) is a well-established protocol. These FPDs require a laboratory phase of fabrication that varies from days to months ${ }^{4}$ Interim crowns and fixed partial dentures are an important components of fixed prosthodontics treatment. Interim restorations should have sufficient mechanical property to support functional and removable forces, that maintain tooth position and exhibit marginal integrity and biological properties conductive to periodontal health. ${ }^{5}$ The fracture toughness of a material represents its ability to resist crack propagation. This parameter is important in assessing the mechanical strength and long term clinical performance ${ }^{6}$.

Polymethylmethacrylate (PMMA) is the most common material used to fabricate interim FPDs. Thorough extensive research has been done regarding the fracture resistance of variable available provisional materials, there is paucity of information in the literature regarding the flexural strength of provisional materials in vivo condition ${ }^{2,7}$. Hence this study was undertaken to evaluate fracture resistance of two most commonly used provisional material.

Materials used in this study -

\section{Material And Methods}

1. Autopolymerising acrylic resin (DPI)

2. Heat cured acrylic resin (DPI)

3. Modelling wax (Link, MDM Corporation, New Delhi)

4. Type II gypsum product (Kalabhai Karson Pvt Ltd, Mumbai)

5. Cold Mould Seal (DPI, Dental materials, Mumbai)

6. Artificial saliva.

Equipment used in this study:

1. Digital Acrylizer

2. Hydraulic press

3. Universal testing machine.

4. Varsity pattern Dental flasks and clamps.

Sample preparation required preparation of two piece metal mould. Preparation of two piece metal mould for obtaining wax pattern:

A metallic mould was fabricated with 3 metallic plates. The plate in the center had five slot of dimensions of $65 \mathrm{~mm}$ in length $20 \mathrm{~mm}$ in width and $3 \mathrm{~mm}$ in thickness in which the materials were filled to get samples of similar dimensions as in Fig. 1and 2. 


\section{Preparation Of Samples}

The provisional crown material mixed according to manufacturer's instructions, injected into metallic mould and held under compression. The autopolymerising material was retrieved from the mould after complete curing of material. Heat cured material was kept in mould and cured as per manufacturers guidelines as shown in fig 3. Excess material was trimmed by using fine abrasive paper. Dimensions was confirmed using vernier calliper.

\section{Study Design \\ This study was designed in two stages.}

15 samples of each material with dimensions of $65 \times 20 \times 3 \mathrm{~mm}$ were prepared in similar way. Out of 15 samples, 7 samples of each material were stored in room temperature for 24 hours. Remaining 8 samples were stored in artificial saliva under normal atmospheric pressure to stimulate in vivo condition as shown in Fig. 5

\section{Observations \& results}

The load causing fracture was recorded. All the data was recorded, tabulated and subjected to statistical analysis. The mean and standard deviation for each group were determined. The data was determined by using one way ANOVA test.

\section{Evaluation of flexural strength:}

Three point flexural tests were carried out for all the samples in universal testing machine.(Instron Corporation, model 4204,canton mass) as shown in Fig. 6

The flexural load was calculated by using formula -

$\mathrm{S}=3 \mathrm{pl} / 2 \mathrm{wt} 2$

Where,

$$
\begin{aligned}
& \mathrm{p}=\text { applied load, } \\
& \mathrm{l}=\text { span in } \mathrm{mm}, \\
& \mathrm{w}=\text { width in }(\mathrm{m}), \\
& \mathrm{t}=\text { thickness }
\end{aligned}
$$

Result were obtained and graph were plotted as Graph 1 and Graph 2.Significant value of F obtained as in "Table 1."

\section{Discussion}

Contemporary provisional restorative materials have traditionally been resin based. Autopolymerising resin has been in routine use since 1930 and remains the most frequently used material ${ }^{4}$. In spite of best efforts in designing and material modification, failure is there leading to discomfort and time loss. Thus fracture resistance of interim FPD material is an important factor and should be considered prior to selecting a provisional material for the clinical success ${ }^{7}$.

Various studies have been described in literature for comparing the fracture resistance of Heat cure and autopolymerising resin ${ }^{6,7}$. This study was undertaken to evaluate the fracture resistance of heat cure and autopolymerising resin in simulated an in vivo condition. In our study, samples of $65 \times 20 \times 3 \mathrm{~mm}$ in size of each material were prepared by injecting material in mould and materials were cured according to manufacturer's guidelines. After curing materials were stored in custom made artificial saliva for 24 hours and 7 days. These rectangular samples were loaded on a universal testing machine and values at which each sample fractured was noted down. Result obtained after 24 hours and 7 days, showed heat cure resin having higher fracture resistance. This can be explained by difference in method of curing mechanism. However, more investigations can be carried out with use of heat cure resin in long span in vivo condition. This results may provide a rational clinical protocol for fabrication of heat cure resin, Fixed partial denture for long span in vivo condition.

\section{Conclusion}

Within the limitations of the study following conclusions were drawn:

1. Heat cured acrylic resin showed higher flexural strength after 24 hours and 7 days.

2. Flexural strength of autopolymerising resin and heat cured resin not very significantly changed after storing in artificial saliva for 7 days.

\section{Limitations}

All the specimens were manufactured in similar way, although finishing and polishing may affect the specimen. 


\section{References}

[1]. Federick DR. The provisional fixed partial denture. J prosthet Dent 1975.

[2]. Haselton DR, Diaz-Arnold AM, Vargas MA, Flexural strength of provisional Crown and Fixed partial denture, J Prosthet Dent 2002.

[3]. Tacir IH, Kama JD, Flexural properties of glass fiber reinforced acrylic fiber reinforced. Aust dent J 2006,

[4]. Col m viswambaran ,Manjeet kumar an evaluation of fracture resistance of interim fixed partial denture fabricated using polymethymethacrylate and reinforced by different fibres for its optimal placement, MJAFI Vol 67, no4 (2011)

[5]. Lisa A knobloch, ronold e kerrby Relative fracture toughness of acrylic resin, J Prosthet Dent (2012)

[6]. Vachan Poonaja, Seema Poonaja, Rohit Raghavan In vitro comparison of flexure strength and elastic modulus of three provisional crown materials in fixed prosthodontics. Journal of clinical and experimental dentistry.(2013)

[7]. Liju Jacob, Kamalkanth Shenoy, Sanath Shetty flexure strength and hardness of resins for interim fixed partial dentures. (2015)

[8]. PHILIP'S science of Dental materials $11^{\text {th }}$ edi. Page no 150.

[9]. John F. McCabe, Applied Dental Materials , 9 th Edi.

[10]. Robert G Craig, Willium O’Brien Dental materials: properties and Manipulation.

Graph 1: Mean flexural strength values of two material at two different time intervals

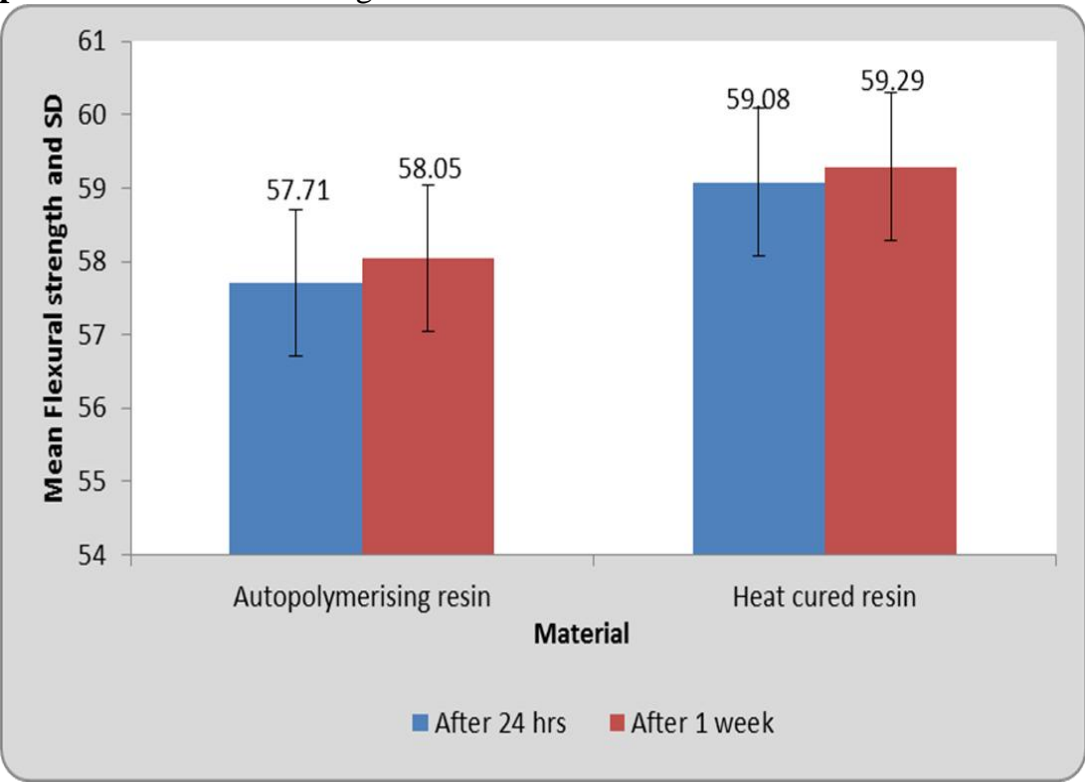

Tabulated results of repeated measure ANOVA for mean flexural strength values of two materials at two time intervals.

Graph 2: Mean flexural strength values of two materials -

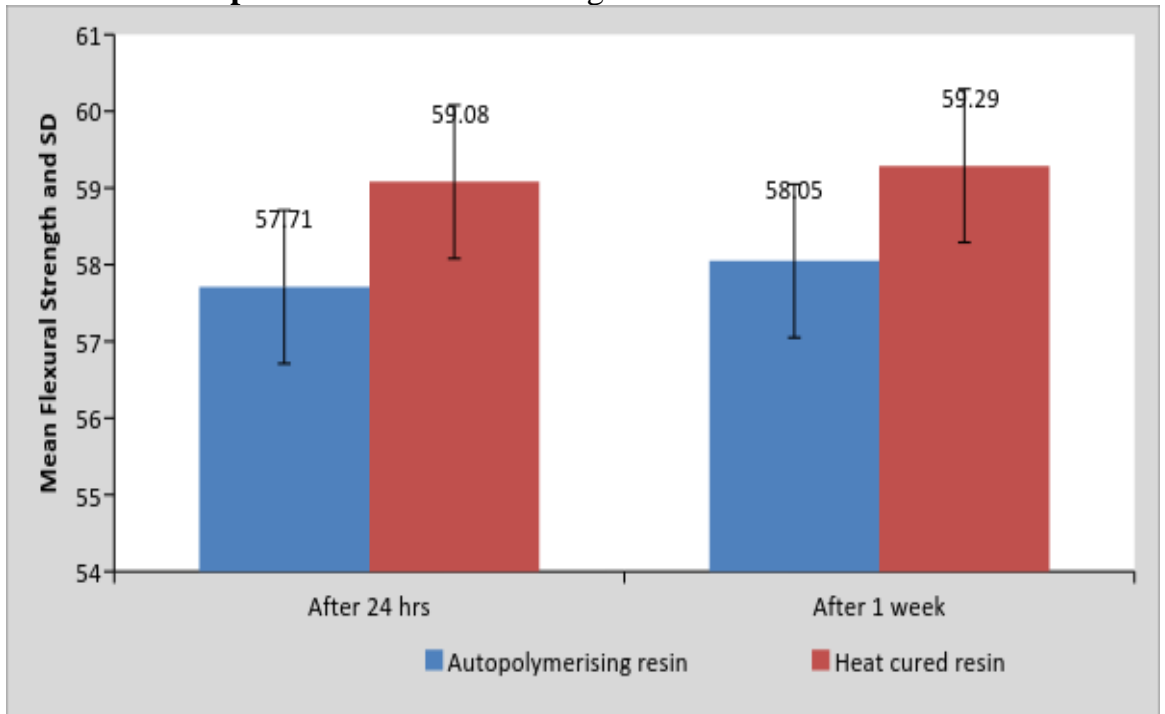

By using student's unpaired t test statistically significant difference was found in mean flexural strength after 24 hours in both the materials $(\mathrm{t}=2.632, \mathrm{p}$-value $=0.014)$. 
Table 1:

\begin{tabular}{|l|c|c|c|c|c|}
\hline \multicolumn{1}{|c|}{ Source of variation } & Sum of squares & df & $\begin{array}{c}\text { Mean } \\
\text { square }\end{array}$ & F-value & p-value \\
\hline Change & 25.70 & 1 & 25.70 & 353.5 & $0.0001, \mathrm{~S}$ \\
\hline Material & 106.5 & 14 & 7.61 & 104.7 & $0.0001, \mathrm{~S}$ \\
\hline Change*Material & 10.09 & 14 & 0.7206 & 9.610 & $0.0001, \mathrm{~S}$ \\
\hline Error(change) & 2.181 & 30 & 0.072 & & \\
\hline
\end{tabular}

When repeated measure ANOVA was applied to mean flexural strength values of 2 different materials at two different storage intervals a significant $F$-value was observed( $F=9.610, p$-value $=0.0001)$ verifying change in the flexural strength with respect to individual materials.

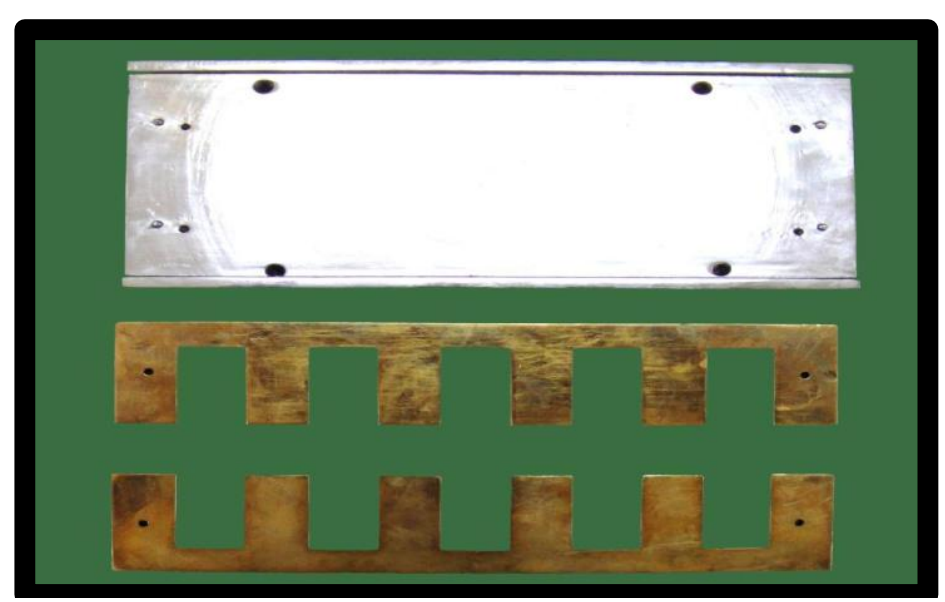

Figure 1 Two piece metal mould.

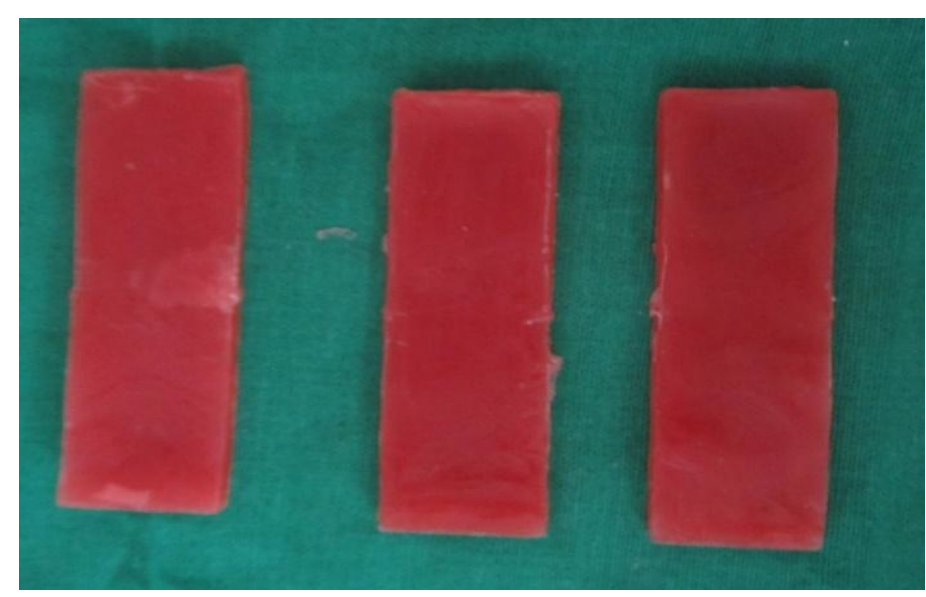

Figure 2 Preparation of samples.

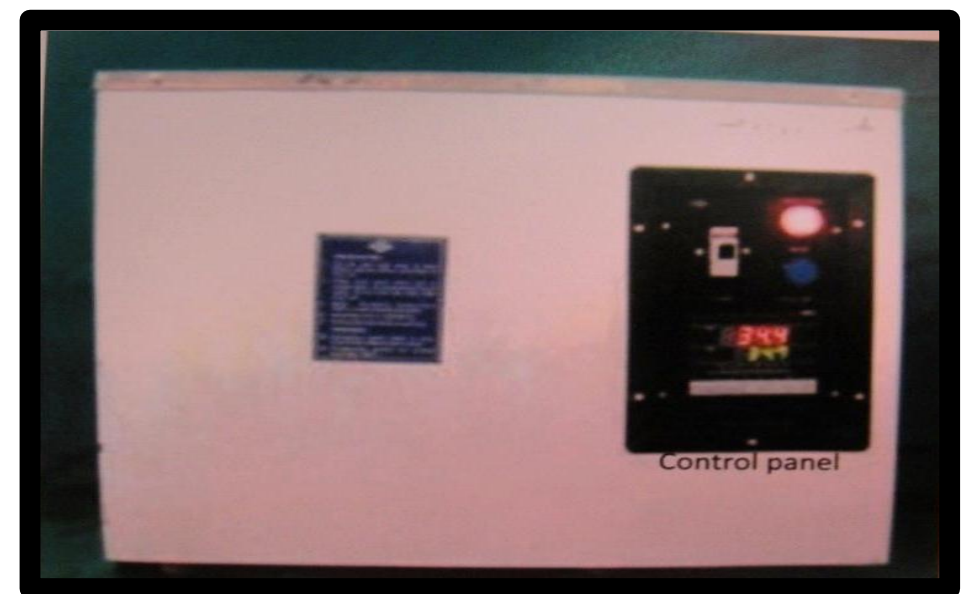

Figure 3 Digital Acrylizer. 


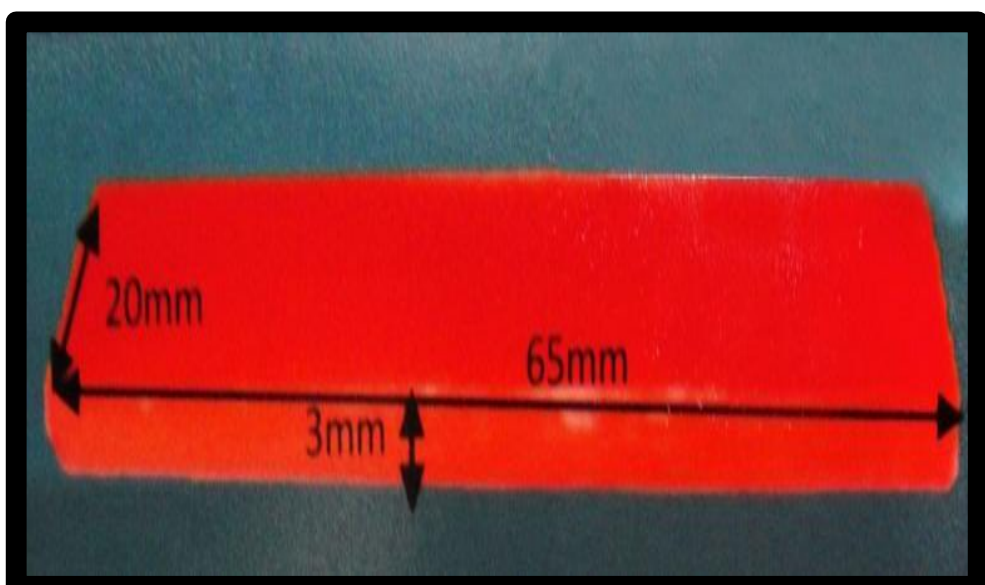

Figure 4 Wax pattern of Sample.

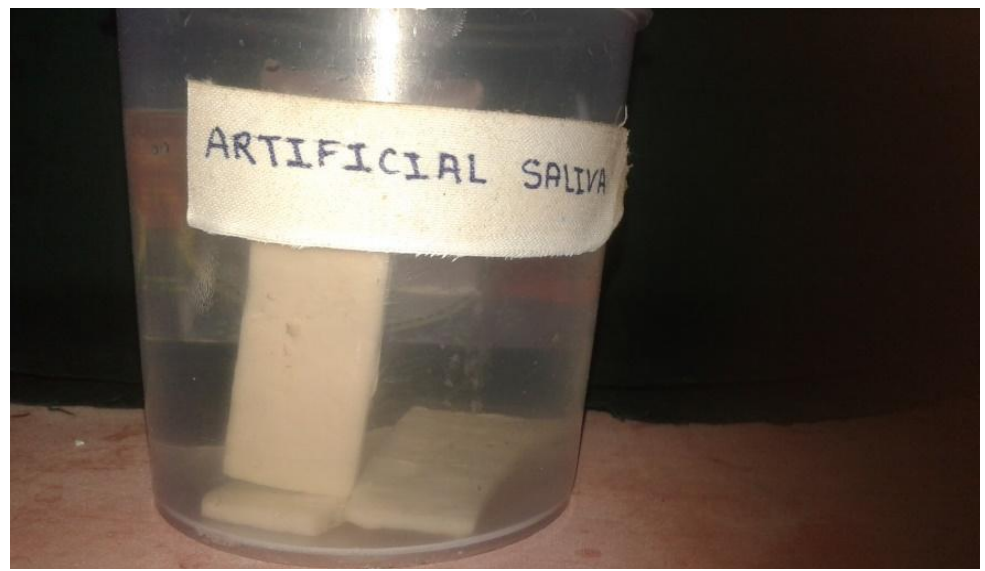

Figure 5: Samples stored in artificial saliva.

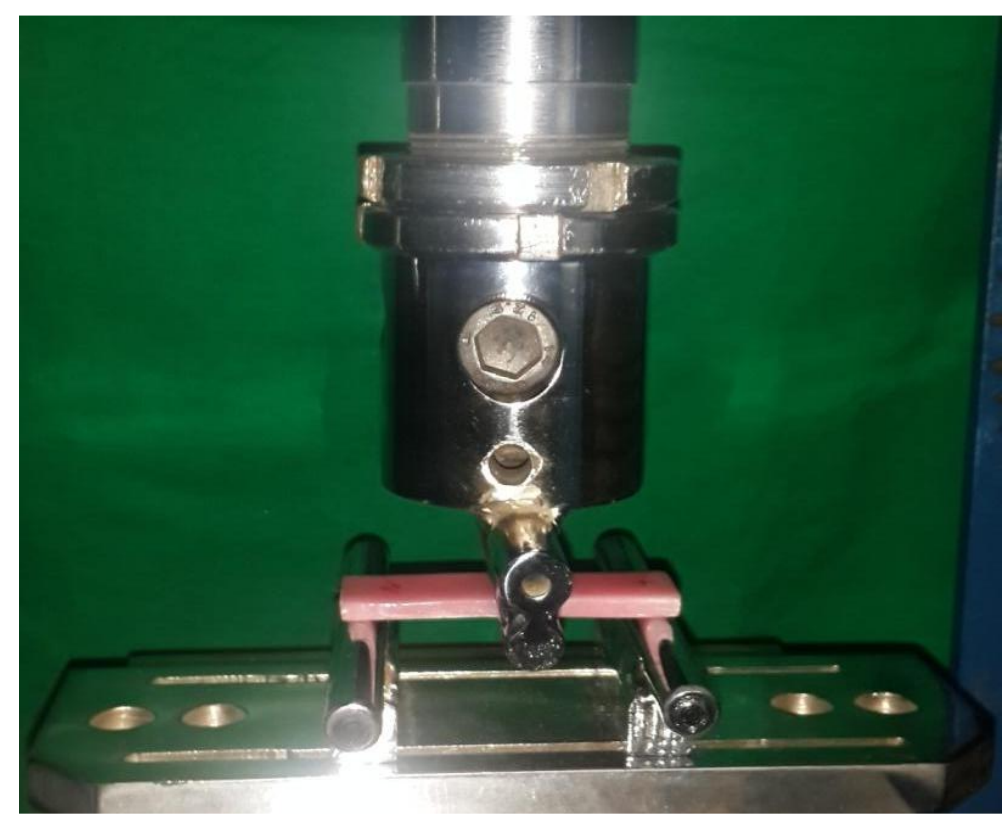

Figure 6 : Acrylic samples testing on Universal testing machine. 